\title{
Estilos de vida promotores de salud en mujeres de los programas sociales de Villavicencio-Colombia
}

\author{
The lifestyles of people attached to Villavicencio's social \\ programmes working as women's health promoters
}

\section{Estilos de vida dos promotores de saúde em programas sociais das mulheres Villavicencio-Colômbia}

\author{
Emilce Salamanca-Ramos ${ }^{1}$ \\ 1 Enfermera, MSc, Docente, Facultad de Ciencias de la Salud, Universidad de los Llanos, Villavicencio, Colombia. \\ Estudiante Doctorado en Ciencias Sociales, Niñez y Juventud \\ Email: emilcesalamanca@gmail.com
}

Recibido: agosto 03 de 2014

Aceptado: agosto 25 de 2015

\begin{abstract}
Resumen
Las enfermedades crónicas no transmisibles han generado y siguen afectando la salud pública de los países en el mundo. La promoción de la salud como estrategia para la prevención de enfermedades no transmisibles se ha fortalecido en las instituciones y organizaciones de salud para el control de las mismas. Conocer los patrones de comportamiento de las personas frente a la salud, en especial en las mujeres, que juegan un papel transcendente en la educación y hábitos de estilos de vida saludable. El objetivo fue determinar el perfil de estilos de vida promotores de salud en las mujeres adscritas a los programas del Departamento de la Prosperidad Social - D.P.S. del municipio de Villavicencio, Colombia. Estudio descriptivo, cuantitativo. La población estuvo constituida por 4.518 mujeres y una muestra de 354. Se utilizó el Instrumento HPLP-II (Perfil de estilos de vida promotor de la salud) (Pender y Walker -1996) para la recolección de la información. Los resultados señalan que los estilos de vida promotores de salud los valores que más predominan están entre 112 y 164 puntos, con una frecuencia de $78,8 \%$, con una media de es 2,5 manteniéndose una homogeneidad en las dimensiones, a excepción de la sub escala de actividad física, con media de 1,86.

Es importante la intervención de programas de salud y educación que fomente los estilos de vida salud en esta población, en especial cuando las mujeres son líderes en la comunidad y en sus familias. También, se visualiza que el aspecto que necesita mayor fortalecimiento es la actividad física.
\end{abstract}

Palabras clave: promoción de la salud, estilo de vida, prevención de enfermedades. (Fuente: DeCS, BIREME. Versión 06 2014)

\begin{abstract}
Chronic non-communicable diseases (CNCD) have caused concern for and continue to affect the public health of countries all around the world. Promoting health as a strategy for preventing NCDs has been strengthened in the institutions and health organisations for controlling them, aimed at understanding people's behaviour patterns regarding health, especially women, who play a transcendent role in education and inculcating healthy lifestyle habits. This work was thus aimed at determining the lifestyle profiles of people attached to Villavicencio's Social Prosperity Department's (Departamento de
\end{abstract}


Prosperidad Social - DPS) social programmes working as women's health promoters. This was a quantitative descriptive study; the population consisted of 4,518 women and sample of 354. The Health-Promoting Lifestyle Profile (HPLP-II) (Pender and Walker, 1996) was used for collecting the information; the resulting data led to a score of 112 to 164 points regarding health promoters' lifestyles (78.8\% frequency, 2.5 mean), homogeneity of dimensions being maintained, except for the physical activity subscale (1.86 mean).

Health and education programmes promoting healthy lifestyles in this population must be encouraged, especially when women are leaders in the community and in their families. The work also raised awareness of physical activity being the aspect which needs further strengthening.

Key words: Health promotion, lifestyle, disease prevention (source: DeCS, BIREME. Version 06 - 2014).

\section{Resumo}

De doenças crônicas não transmissíveis geraram e continuam a afectar a saúde dos países do mundo. A promoção da saúde como uma estratégia de prevenção de doenças não transmissíveis foi reforçada ao longo de todas as instituições e organizações de saúde para controlá-los. Conhecer os padrões de comportamento dos indivíduos em relação à saúde, especialmente em mulheres, que desempenham um papel transcendental na educação e hábitos de vida saudáveis. O objetivo é determinar o perfil dos estilos de vida de promoção da saúde em mulheres atribuídas aos programas do Departamento de prosperidade social - DPS da cidade de Villavicencio, Colômbia. Estudo descritivo e quantitativo. A população era constituída por 4,518 mulheres e uma amostra de 354. O instrumento Pender HPLP-II (promotor de estilos de vida de seu perfil de saúde) (Pender y Walker -1996) foram usados para a recolha de informações. Os resultados indicam que os estilos de vida promotores de valores de saúde que predominam mais entre 112 e 164 pontos, com uma taxa de $78,8 \%$, com uma média de é 2.5 mantendo uma homogeneidade das dimensões, com excepção da escala de sub de atividade física, com média de 1,86.

É importante a intervenção dos programas de educação e saúde que incentivam a saúde de estilos de vida nessa população, especialmente quando as mulheres são líderes na Comunidade e suas famílias. Além disso, exibe o aspecto que precisa reforçar é a atividade física.

Palavras-chave: promoção da saúde, estilos de vida saudáveis, a prevenção da doença. (Fonte: DeCS, BIREME. Versão 06 - 2014).

\section{Introducción}

La salud y la enfermedad son procesos relacionados que dependen de la influencia de múltiples factores que interaccionan, entre ellos sobresalen aquellos que operan sobre el conjunto de la población. El ámbito de estos determinantes permite plantear las diversas intervenciones de promoción de la salud y prevención de la enfermedad (Hernández-Agudelo, 2011). La promoción de la salud se consigue a través de tres mecanismos intrínsecos o internos de las personas, según lo planteado por la Organización Panamericana de la Salud (OPS); estos son: El auto cuidado, es decir, las decisiones y acciones que la persona lleva a cabo en beneficio de su propia salud. La ayuda mutua o acciones que las personas realizan para ayudarse unas a otras de acuerdo con la situación que estén viviendo. Los entornos sanos o creación de las condiciones ambientales que favorecen la salud, tales como las escuelas saludables que se constituyen en escenarios potenciadores de las capacidades de los niños y las niñas, en las que la educación para la salud y la promoción de la salud son opciones pedagógicas para el desarrollo humano y la formación en valores (Giraldo, 2010).

El Modelo de Promoción de la Salud (MPS) propuesto por Nola Pender se utiliza para examinar e intervenir los estilos de vida que promueven la salud (Chilton et al. 2006); ha servido como marco para investigaciones destinadas a predecir sistemas de promoción de la salud globales y conductas específicas como la del ejercicio físico: entre otros. Según Pender (1990), los determinantes de los estilos de vida y la promoción de la salud están establecidos por las características y las experiencias individuales, los conocimientos y efectos relativos de la conducta, así como la probabilidad de participar o no en comportamientos que promueven la salud. El MPS como referente teórico de la investigación, ayudó a identificar los comportamientos que tienen las mujeres frentes a los estilos de vida. Es uno de los modelos más predominantes en la promoción de la salud en enfermería (Giraldo, 2010) y uno de los más utilizado en el control y prevención de las enfermedades crónicas no transmisibles.

Las enfermedades crónicas no transmisibles (ECNT) representan con diferencia la causa de defunción más importante en el mundo, pues acaparan un $63 \%$ del número total de muertes anuales. El $80 \%$ de las ECNT se dan en los países de ingresos bajos y medios. Más de 9 millones de las muertes atribuibles a las ECNT se producen en personas menores de 60 años. En términos mundiales, las ECNT afectan por igual a hombres y mujeres. Estas enfermedades comparten factores de 
riesgo comunes que incluyen el tabaquismo, la inactividad física, el uso nocivo del alcohol y la dieta no saludable; por ejemplo. En 2008, 1500 millones de adultos (de 20 y más años) tenían sobrepeso, mientras alrededor de 43 millones de niños menores de cinco años tenían sobrepeso en 2010 (O.M.S, 2013). En Latinoamérica y en países como Colombia, las enfermedades crónicas se han convertido en un grave problema de salud pública, al ocupar los primeros lugares de morbilidad y mortalidad en los perfiles epidemiológicos de los países (Bonilla-Chacìn, 2012).

En Colombia, el $76 \%$ de la morbilidad es ocasionada por las enfermedades crónicas no transmisibles, porcentaje similar al de los países desarrollados. Hay una prevalencia alta de la hipertensión arterial con un $22,8 \%$, asociada con el aumento de riesgo para cardiopatía isquémica en un $49 \%$, y el $62 \%$ de los trastornos cerebro vasculares. Por otro lado, la diabetes aumentó del 2,0\% en la población adulta de 1999 hasta un $2,6 \%$ en 2007 , con un $19,8 \%$ de discapacitados diabéticos que es 2,5 veces superior a la prevalencia de discapacidad estimada para la población general no diabética, con mayor afectación por grupos de edad entre los 30 y 39 años con el 25\% (PDSP, 2013).

Las ECNT se pueden prevenir y controlar por medio de cambios en el estilo de vida, políticas públicas e intervenciones de salud, y requieren un abordaje intersectorial e integrado. De eliminarse los principales factores de riesgo para el padecimiento de enfermedades crónicas, se podrían prevenir cerca de tres cuartas partes de la carga atribuible a las enfermedades cardiovasculares, los accidentes cerebro vasculares y la diabetes de tipo 2, y un $40 \%$ de los casos de cáncer (O.M.S., 2011). Una de las estrategias para la prevención y el control de estas enfermedades es el énfasis en la promoción de la salud, la educación y la prevención, al igual que en la detección temprana, el tratamiento oportuno y la calidad de la atención para las personas que ya tienen alguna ECNT o que muestran señales de alerta por la presencia de factores de riesgo (O.P.S., 2012).

EI MPS propuesto ofrece la posibilidad de valoración de los estilos de vida de las personas que tienen riesgo de sufrir una alteración de la salud; para este caso, son las mujeres líderes de los programas sociales, y actuar antes de que se produzcan alteraciones que lleven a una demanda hospitalaria con riesgo para la vida y su funcionalidad. En este contexto, el interés de esta investigación es determinar los perfiles de los estilos de vida promotor de la salud que tienen las mujeres del municipio de Villavicencio, en especial aquellas que lideran procesos comunitarios en sus comunas y tam- bién en sus familias, a través de este instrumento que surge del modelo de la promoción de la salud.

\section{Materiales y métodos}

Estudio descriptivo, cuantitativo. La población estuvo constituida por 4.518 mujeres inscritas en los Programas del Departamento de la Prosperidad Social (D.P.S.) del municipio de Villavicencio. La muestra fue probabilística estratificada por comuna, por asignación óptima o de Neyman, con un nivel de confiabilidad del $95 \%$ y un error máximo admisible de 5\%, determinándo; el tamaño de la muestra que corresponde a 354 mujeres. Se usa este diseño de muestra y se tomó como variable la ubicación de la líder por comuna, puesto que existen marcadas diferencias en la variabilidad de las observaciones dentro de los estratos socioeconómicos; en este caso; la ubicación de las mujeres en las comunas. Además de tener en cuenta el tamaño de los estratos, se tuvo en cuenta la dispersión de los datos dentro de cada estrato. De ésta manera se obtiene una muestra más grande de aquellos estratos que sean más heterogéneos, y los elementos de la población tienen una misma probabilidad de ser seleccionados.

La unidad de observación se conformó con las mujeres adscritas a los Programas del D.P.S., con los siguiente criterios de inclusión: mayor de 18 años, inscrita activamente en el programa presidencial de Acción Social del municipio de Villavicencio, vivir en el municipio de Villavicencio y aceptar el consentimiento informado. La información se obtuvo a través de dos instrumentos: Un formulario tipo encuesta para documentar características socio-demográfico de las mujeres. Un segundo cuestionario fue The Health-Promoting Lifestyle Profile II (HPLP II) en versión español (Walker et al., 1990) (Pender et al., 1990).

El HPLP II fue validado en versión en inglés y español a nivel internacional, y reportó alfas de Cronbach (versión en inglés) del total de HPLP II, 0,94 (Stuifbergen y Roberts, 1997), versión en español con varianza de $45,9 \%$ y el coeficiente de confiabilidad alfa para la escala total fue de 0,93 y 2 semanas de la fiabilidad testretest fue de 0,86; los coeficientes alfa para las sub escalas oscilaron entre 0,70 hasta 0,87 (Walker et al., 1990). Un estudio similar en población latinoamericana produjo como coeficiente de confiabilidad de alfa para la escala total fue de 0.923 (Vidal et al., 2014) y 0,93 (Laguado y Gómez, 2014), congruente en estudios de validación inicial del instrumento.

El instrumento HPLP II se utiliza para examinar la percepción de control de la salud (Walker et al., 1990) 
(Walker et al., 1996); está conformado por 52 ítems agrupados en seis dimensiones: responsabilidad de la salud, actividad física, nutrición, crecimiento espiritual, relaciones interpersonales, manejo del estrés. Es valorado a través de la escala tipo Likert: $\operatorname{Nunca}(\mathrm{N})=1$, algunas veces $(A)=2$, frecuentemente $(M)=3$, Rutinariamente $(R)=4$; con valoración mínima de 52 y máxima de 208; la sumatoria de los ítems da una valoración de cada dimensión, con una mayor puntuación indica un nivel más alto de estilos de vida promotores de salud. Para el procesamiento de la información se utilizó el paquete estadístico SPSS ${ }^{\circ}$ (Statistical Product and Service Solutions) versión 14.0.

Se tuvieron en cuenta los parámetros éticos para estudios con seres humanos contenidos en la Resolución 08430 de 1993 del Ministerio de Salud de Colombia y el Código de Ética de Enfermería que se establece en la ley 911 de 2004; además, se aplicaron los principios de confidencialidad, veracidad y beneficencia y no maleficencia. Para ello, se realizó el consentimiento informado previo a la aplicación del instrumento, para garantizar la libre participación en el estudio. Además, la investigación fue regulada por el Comité de Bioética de la Universidad de los Llanos (UNILLANOS, 2010).

\section{Resultados y discusión}

La población se caracterizó por estar en edad productiva, cuya edad media es de 34 años con una desviación estándar de 10,1, lo que implica que el $68 \%$ de la población se encuentra entre 24 y 44 años de edad. En lo relacionado con la vivienda, el $66,4 \%$ vive. en condiciones de arriendo o con sus familiares. En cuanto a nivel educativo, el $27,7 \%$ con primaria completa, el $23,2 \%$ secundaria completa; llama la atención que un $2,3 \%$ no tenga nivel educativo alguno; de otro lado, se destaca el nivel técnico en jóvenes. El estado civil de las mujeres en estudio es, en su mayoría, la unión libre $(38,4 \%)$ superando en un $10 \%$ a las casadas, cifra superior a la reportada por la encuesta nacional de demografía y salud con el 33,3\% (Profamilia, 2010); esto podría estar relacionado con un crecimiento paulatino en la unión marital de hecho en esta población.

De las mujeres estudiadas, un $8 \%$ no tiene hijos. El cuanto al número de hijos, no se encuentra una diferencia significativa respecto a lo reportado en la ENDS 2010: el 43,2\% tiene entre 3 y 4 hijos; en el estudio fue de $44,9 \%$ (Tabla 1). Colombia vive un proceso de plena transición demográfica, con una natalidad descendente en todos los grupos de mujeres en edad fértil, de los 15 a los 49 años, con una reducción constante del índice sintético de fecundidad, se acerca cada vez más al nivel de remplazo, con 2,1, en la ENDS realizada en 2010, pero con una resistencia a la baja en la fecundidad en adolescentes (PDSP, 2013).

Los estilos promotores de salud en la población, se encontró que el nivel predominante es el medio, con valores entre 112 y 164 puntos, con un $78,8 \%$, si se tienen en cuenta que el valor máximo en el total de la valoración del instrumento es de 208. El valor minino de la población estudiada estuvo en 83 y el máximo 184. La escala baja corresponde al $17,2 \%$ de la población. Al explorar por cada uno de los programas que desarrolla el D.P.S, se encontró que el $4 \%$ de la valoración alta estaba en el programa "Más familias en acción" (Tabla 2).

Se observó que la media global de los estilos promotores de salud es 2,5 manteniendo una homogeneidad entres las dimensiones, a excepción de la sub escala de actividad física, con media de 1,86 (Tabla 3). Estudios con mujeres en población chilena reportaron una media de 2,57 y la más alta de la sub escala es el crecimiento espiritual con una media de 3,22 (Vidal et al., 2014). Sin embargo, las sub escalas de relaciones interpersonales y crecimiento espiritual son muy similares; estudios afines indican que relaciones estrechas entre ambas implican un sentido de conexión y pertenencia (Carreno et al, 2006), (Carson, 2000). Esto quiere decir que las mujeres estudiadas establecen buenas relaciones personales, que permiten transcender más allá de lo individual para pensar en lo colectivo.

En el crecimiento espiritual, dado que la media es de 2,97 y la desviación estándar es de 0,07, implica que un $68 \%$ de la población se ubicaría en una escala de percepción entre 2,9 y 3,04, que sería más cercano a una escala de: frecuentemente. Lo contrario ocurre con la dimensión de actividad física, que muestra una media de 1,86 y una desviación estándar de que no alcanza a la escala de: a veces. Esta cifra exige un esfuerzo mayor de los programas de promoción de la salud, para comprender las dinámicas, situaciones y factores que inciden en esta dimensión. Estudios con población colombiana, que ha explorado esta dimensión, han mostrado también una similitud: un $51 \%$ obtuvo por debajo de la media para ese momento (Triviño et al., 2007). Al interior de la sub-escala, los ítems más bajos en el promedio de la HPHL II fueron "sigue programas de ejercicio planificados" (45,5\%), "examina su pulso cuando está haciendo ejercicio" (54,5\%) y "alcanzo el pulso cardiaco objetivo cuando hace ejercicio" $(56,2 \%)$.

En Colombia, solamente el $26 \%$ de la población cumple con el mínimo de actividad física recomendada en el grupo de edad de 13 a 17 años; este porcentaje 
Tabla 1. Característica sociodemográficas de las mujeres del D.P.S. $\mathrm{N}=354$

\begin{tabular}{|c|c|c|}
\hline Edad & Fr & $\%$ \\
\hline Entre 18 - 27 años & 97 & 27,4 \\
\hline De 2 - 36 años & 110 & 31,1 \\
\hline De 36 - 45 años & 97 & 27,4 \\
\hline De 45 - 54 años & 33 & 9,3 \\
\hline De 54 - 64 años & 17 & 4,8 \\
\hline Media & & 34,81 \\
\hline Desv. Típica & & 10,11 \\
\hline \multicolumn{3}{|l|}{ Tenencia de Vivienda } \\
\hline Propia & 112 & 31,6 \\
\hline Arriendo & 170 & 48,0 \\
\hline Familiar & 65 & 18,4 \\
\hline NR & 7 & 2,0 \\
\hline \multicolumn{3}{|l|}{ Nivel de Estudios } \\
\hline Sin estudios & 8 & 2,3 \\
\hline Primaria incompleta & 57 & 16,1 \\
\hline Primaria completa & 98 & 27,7 \\
\hline Secundaria incompleta & 63 & 17,8 \\
\hline Secundaria completa & 82 & 23,2 \\
\hline Técnico & 40 & 11,3 \\
\hline Universitario incompleto & 3 & 8 \\
\hline Universitario completo & 3 & 8 \\
\hline \multicolumn{3}{|l|}{ Estado Civil } \\
\hline Soltero & 78 & 22,0 \\
\hline Casado & 99 & 28,0 \\
\hline Separado & 22 & 6,2 \\
\hline Viudo & 19 & 5,4 \\
\hline Unión marital de hecho & 136 & 38,4 \\
\hline \multicolumn{3}{|l|}{ Número de hijos } \\
\hline Sin hijos & 28 & 7,9 \\
\hline 1-2 Hijos & 112 & 31,6 \\
\hline 3-4 Hijos & 159 & 44,9 \\
\hline 5-6 Hijos & 38 & 10,7 \\
\hline 7-8 Hijos & 12 & 3,4 \\
\hline Más de 10 Hijos & 5 & 1,4 \\
\hline
\end{tabular}

aumenta a $42,6 \%$ entre los 18 y 64 años; sin embargo, llama la atención que la actividad física en tiempo libre solamente fue practicada por el $8,6 \%$ de la población entre 13 y 17 años y en un 5,9\% entre 18 y 64 años (Ministerio de salud de Colombia, 2012). En cuanto a la prevalencia de actividad física, se reporta que muy bajos porcentajes de la población realizan actividad física, lo que se convierte en objetivo de obligatorio abordaje desde los entes nacionales e internacionales relacionados con la práctica de actividad física, a través de estrategias o intervenciones efectivas que permita mejorar la calidad de vida (Vidarte et al., 2011). La actividad física es un factor protector de salud que tiene repercusiones positivas en la Salud Pública, de tal manera que sea un espacio de empoderamiento entre las acciones de salud y educación en beneficio de un colectivo.

La concentración de la población se situó en un estilo de vida promotor de salud moderadamente adecuado; esto significa que los programas del D.P.S. y los entes territoriales encargados de esta población podrían intensificar las acciones dirigidas a la promoción de la salud que generen impacto a la población y, así, evitar que se presenten factores de riesgo para las enfermedades crónicas no transmisibles (ECNT). Iguales resultados se encontraron en poblaciones latinoamericanas (Cid et al., 2006) Centro America (Reséndiz et al., 2010) y otros continentes (Hosseini, et al., 2010), en las que los estilos de vida de las personas comprenden conductas negativas para la salud, las cuales se pueden evitar mediante la adopción de conductas protectoras.

\section{Conclusiones}

Al explorar los estilos de vida promotores de salud, dentro del modelo de la promoción de la salud que plantea Nola Pende, las mujeres del estudio no mostraron una satisfactoria conducta para fomentar el bienestar y cuidado de la salud como líderes de los programas de D.P.S. y como mujeres cabeza de hogar. Esta situación llama la atención debido a que los programas que maneja el Estado están muy centrados en la política de articular acciones de beneficios con programas de salud y, en especial, con acciones de promoción de la salud.

El estilo de vida que promueve la salud de las mujeres líderes de los programas sociales está en el nivel medio y el nivel de crecimiento espiritual es el más alto, seguido por las relaciones interpersonales, la nutrición, el manejo del estrés y la responsabilidad de la salud; el nivel más bajo fue la actividad física.

Es importante la articulación de acciones del DPS con las instituciones de salud y otros programas interesados en promover trabajos que fomenten los estilos de vida saludables y mejoren la calidad de las mujeres. A través del aumento de la actividad física, el manejo del estrés y un compromiso mayor en la responsabilidad de la salud, se puede reducir sustancialmente la carga de las enfermedades crónica no transmisibles en esta población. 
Tabla 2. Estilos de vida promotores de salud, por programa. $N=354$

\begin{tabular}{|l|c|c|c|c|c|c|c|c|}
\hline \multirow{2}{*}{\multicolumn{1}{|c|}{ Programa }} & \multicolumn{2}{|c|}{ Bajo } & \multicolumn{2}{c|}{ Medio } & \multicolumn{2}{c|}{ Alto } & \multicolumn{2}{c|}{ Total } \\
\cline { 2 - 11 } & $\mathbf{N}$ & $\%$ & $\mathbf{N}$ & $\%$ & $\mathbf{N}$ & $\%$ & $\mathbf{N}$ & $\%$ \\
\hline Más familias en acción & 22 & 6,20 & 188 & 53,10 & 13 & 3,7 & 223 & 63 \\
\hline Madres líderes & 36 & 10,16 & 48 & 13,60 & 1 & 0,3 & 85 & 24 \\
\hline Jóvenes en acción & 2 & 0,56 & 22 & 6,20 & 0 & 0 & 24 & 7 \\
\hline Mujeres ahorradoras & 1 & 0,28 & 21 & 5,93 & 0 & 0 & 22 & 6 \\
\hline Total & 61 & 17,2 & 279 & 78,8 & 14 & 4,0 & 354 & 100 \\
\hline
\end{tabular}

Tabla 3. Estilos de vida promotores global y por dimensiones $\mathrm{N}=354$

\begin{tabular}{|l|c|c|c|}
\hline \multicolumn{1}{|c|}{ Ítem } & $\overline{\mathbf{x}}$ & $\sigma$ & C.V \\
\hline Promotora de la Salud Lifestyle & 2,5 & 0,09 & $3,46 \%$ \\
\hline Crecimiento Espiritual & 2,97 & 0,07 & $2,4 \%$ \\
\hline Relaciones Interpersonales & 2,69 & 0,06 & $2,1 \%$ \\
\hline Nutrición & 2,58 & 0,05 & $1,83 \%$ \\
\hline Manejo del estrés & 2,45 & 0,08 & $3,15 \%$ \\
\hline Responsabilidad de la salud & 2,36 & 0,05 & $2,11 \%$ \\
\hline Actividad Física & 1,86 & 0,06 & $3{ }^{\prime} 3 \%$ \\
\hline
\end{tabular}

Finalmente, enfatizar que la educación en salud fundamental en la modificación de los estilos de vida. El Modelo de la Promoción de Salud sería una herramienta de utilidad para el desarrollo de programas que, junto con los entes teritoriales del municipio y la universidad, puedan ser ofertados en beneficio de grupos poblacionales que lideran procesos sociales importantes en sus comunidades y en sus familias. Una situación como ésta permite modificar las conductas de riesgo en las personas y en los colectivos para la adopción de estilos de vida saludables.

\section{Agradecimiento}

La autora agradece a la Universidad de los Llanos y los estudiantes principiantes de investigaciones del programa de Enfermería por su apoyo en el desarrollo de esta investigación.

\section{Referencias}

Hernández-Agudelo I, Gil de Miguel A, Delgado-Rodríguez M, Bolúmar-Montrull F, Benavides FG, et al., 2011. Manual de Epidemiología y Salud pública para grados en ciencias de la salud.
Segunda edición. Editorial Médica Panamericana, Madrid, p. 400.

Giraldo-Osorio A, Toro-Rosero MY, Macías-Ladino AM, ValenciaGarcés CA, Palacio-Rodriguez S. La promoción de la salud como estrategia para el fomento de estilos de vida saludables. Hacia promoc. Salud [serial onthe Internet]. 2010;15(1):128143.

Chilton L, Hu J, Wallace DC. Health-promoting lifestyle and diabetes knowledge in Hispanic American adults. Home Health Care Management \& Practice. 2006;18(5):378-385. DOI: $10.1177 / 1084822306288059$

O.M.S. Organización Mundial de la Salud. 2013. Los 10 datos sobre enfermedades no transmisibles. Boletín informativo. Bogotá, Colombia.

Bonilla-Chacìn ME. 2012. Promoción de estilos de vida saludable en América Latina y el Caribe. El Banco Mundial. 2012 Mayo. Nueva York, EEUU.

Ministerio de Salud y Protección Social. 2013. Plan de Cenal de Salud Pública 2012 -2021. Bogotá, Colombia.

O.M.S. Organización Mundial de la Salud. 2011. Enfermedades no transmisibles y salud mental. Unidos contra la enfermedades no transmisibles. Bogotá, Colombia.

O.P.S. Organización Panamericana de Salud. 2012. 28.a Conferencia Sanitaria Panamericana 64.a sesión del comité regional. 
Washington, D.C., EUA, del 17 al 21 de septiembre del 2012. Resolución CSP28.R13. Estrategia para la prevención y el control de las enfermedades no transmisibles. Washington, D.C., EUA

Walker SN, Kerr MJ, Pender NJ, Sechrist KR. A Spanish language version of the health-promoting lifestyle profile. Nurs Res. 1990;39(6):268-273.

Pender N, Walker S, Sechrist K, Frank M. Predicting Health-Promoting Lifestyles in the Workplace. Nurs Res. 1990;39(6):326-332.

Stuifbergen AK, Roberts GJ. Health promotion practices of women with multiple sclerosis. Arch Phys Med Rehabil. 1997;78(12 Suppl. 5):3-9. [fecha de acceso 28 de agosto de 2015] URL: http://www.ncbi.nlm.nih.gov/pubmed/9422001.

Laguado E, Gómez MP. Estilos de vida saludable en estudiantes de Enfermería en la Universidad Cooperativa de Colombia. Hacia promoc. Salud. 2014;19(1):68-83.

Walker SN, Hill-Polerecky DM. 1996. Psychometric evaluation of the Health-Promoting Lifestyle Profile II. Unpublishedmanuscript, University of Nebraska Medical Center.

UNILLANOS. Universidad de los Llanos. 2010. Acuerdo Superior No. 012, Comité de Bioética de Universidad de los Llanos. ViIlavicencio, Colombia.

Profamilia - Ministerio de Salud. 2010. Encuesta nacional demografía y salud 2010. Estado conyugal actual. Pág. 159. Bogotá, Colombia.

Vidal-Gutiérrez D, Chamblas-García I, Zavala-Gutiérrez M, MüllerGilchrist R, Rodríguez-Torres MC, Chávez-Montecino A. Determinantes sociales en salud y estilos de vida en población adulta de Concepción, Chile. Ciencias de Enfermería. [revista en la Internet]. 2014 Abr [citado 2014 Sep 02]; 2014;20(1):61-74.

Carreno J, Vyhmeister G, Grau L, Ivanovic D. A health promotion programme in Adventist and non-Adventist women based on Pender's model: a pilot study. Public Health. 2014;20(1):61-74.

Carlson ED. A case study in translation methodology using the Health-Promotion Lifestyle Profile II. Public Health Nursing. 2000;17(1):61-70. doi: 10.1046/j.1525-1446.2000.00061.x

Triviño Z, Stiepovich J, Merino JM. Factores predictores de conductas promotoras de salud en mujeres peri- post-menopáusicas de Cali, Colombia. Revista Colombia Médica. 2007;38(4):395407.

Ministerio de Salud y Protección Social. 2012. Instituto Nacional de Cancerología, ESE, Informe estadístico de las Enfermedades Crónicas No Transmisibles.

Vidarte-Claros JA, Álvarez CV, Sandoval-Cuellar C, Alfonso-Mora ML. Actividad Física: estrategias de promoción de la salud. Hacia la Promoción de la Salud. 2011;16(1):202-218.

Cid HP, Merino EJ, Stiepovich BJ. Factores biológicos y psicosociales predictores del estilo de vida promotor de salud. Rev Méd Chile. 2006;134(12):1491-1499.

Reséndiz E, Aguilera P, Rocher ME. Estilos de vida e índice de masa corporal de una población de adultos del sur de Tamaulipas, México. Aquichán. 2010;10(3):244-252.

Hosseini H, Torkani S, Tavakol K. The effect of community health nurse home visit on self-care self-efficacy of the elderly living in selected Falavarjan villages in Iran in 2010. Iran J Nurs Midw ifery Res. 2013;18(1):47-53. 